\title{
The Importance of Coupling between Thermal and Molar Fluxes in a Nitrogen-Oxygen Distillation Column*
}

\author{
L.V. van der $\operatorname{Ham}^{a, * *}$ and S. Kjelstrup ${ }^{a, b}$ \\ ${ }^{a}$ Department of Chemistry, Norwegian University of Science and Technology, N-7491 Trondheim, Norway \\ ${ }^{b}$ Department of Process and Energy, Delft University of Technology, 2628 CA Delft, The Netherlands \\ E-mails: leen.vanderham@chem.ntnu.no, signe.kjelstrup@chem.ntnu.no
}

\begin{abstract}
A model for the transfer of mass and thermal energy in a vapour-liquid region is used to investigate the influence of neglecting coupling on the transfer rates. As an example, we studied a nitrogen-oxygen distillation column. Using a combination of stage and point boundary conditions, a nitrogen transfer profile is obtained that shows the same trend as a profile based on an equilibrium stage distillation model. The distribution of the total transfer over the two column halves is not in agreement, however. This disagreement can be expected to decrease when the dependency of the vapour film thickness on the vapour flow rate and the vapour viscosity is included in the model. The effect of neglecting coupling on the calculated transfer rates changes along the length of the column. The total effect is considerable and should be taken into account in models for the transfer of mass and thermal energy through an interface.
\end{abstract}

Keywords: Distillation; heat transfer; irreversible thermodynamics; mass transfer.

\section{Introduction}

The energy and exergy efficiencies of conventional distillation columns can be increased by distributing the thermal energy addition and removal over the entire length of the column, instead of concentrating it at the top and the bottom (Fonyó, 1974b,a). When optimizing the efficiencies of such columns, it is essential to model the thermal energy flows inside the column as accurately as possible. According to the theory of irreversible thermodynamics, a thermal flux is not only dependent on a temperature difference, but also on concentration differences. In general, each flux is a linear combination of all driving forces. More details on the theory of irreversible thermodynamics can be found, for example, in the monograph by Kjelstrup \& Bedeaux (2008).

Current non-equilibrium distillation models do not explicitly include the coupling between thermal and mass fluxes (Taylor \& Krishna, 1993). Using an ethanol-water distillation column as case study, De Koeijer \& Kjelstrup showed that coupling can have a considerable effect (De Koeijer \& Kjelstrup, 2004; Kjelstrup \& De Koeijer, 2003). This is confirmed by a recent study by Van der Ham et al. (2010) based on a single point in a cryogenic nitrogen-oxygen column.

\subsection{Objectives}

The aim of the current work is to gain more insight into the influence of coupling between thermal and mass fluxes on the calculated transfer rates in a nitrogen-oxygen distillation column. We investigate how this influence changes along the length of the column. We also provide more premises on the thermodynamic description of the transfer of mass and thermal energy through an interface.

\section{Model of the interface region}

The model we use to characterize the coupled transfer of mass and thermal energy in a vapour-liquid region is described by Van der Ham et al. (2010). In this model, the interface region is located in between the bulk liquid and bulk vapour phases; the conditions at its boundaries are equal to the properties of the adjacent bulk phases. The region itself consists of an interface layer in between a liquid and a vapour film. Both the liquid and the vapour film can be represented by multiple control volumes, but the interface is always given by a single control volume. Figure 1 gives a schematic representation of a system consisting of five control volumes.

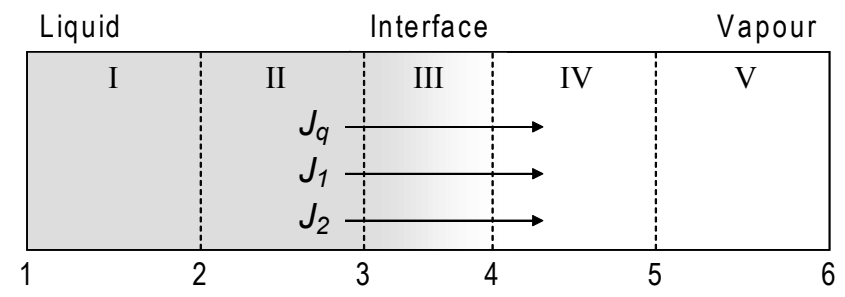

Figure 1. Schematic a vapour-liquid interface region consisting of five control volumes: two liquid volumes (I and II), the vapour-liquid interface (III) and two vapour volumes (IV and V). Point 1 is the liquid boundary of the system and point 6 is its vapour boundary.

The model uses the interface frame of reference, which is also known as the laboratory frame of reference. This corresponds to a system with a non-moving center-of-volume. Using a matrix of total resistivity coefficients, the model can be used to calculate the thermal and molar fluxes for a given 
set of boundary conditions, or driving forces. This section elaborates on the model formulation that was chosen in Van der Ham et al.

\subsection{Choosing the set of fluxes and forces}

The basis of the model is an expression for the local entropy production $\sigma$ of a control volume that is located between points $a$ and $b$. According to the theory of irreversible thermodynamics, the local entropy production is given by the product-sum of conjugate fluxes and driving forces. Different sets of fluxes and driving forces can be used. Bedeaux \& Kjelstrup (2004) describe two possible sets for a system with only thermal and molar fluxes. One can choose to use the total heat flux $J_{q}$ in combination with the chemical potentials $\mu_{j}$ divided by temperature $T$, as shown in Eq. (1), or one can use the measurable heat flux $J_{q}^{\prime}$ in combination with the chemical potentials, evaluated at a constant temperature, divided by the temperature, as shown in Eqs. (2) and (3).

$$
\begin{aligned}
& \sigma=J_{q}\left(\Delta_{a b} \frac{1}{T}\right)+\sum_{j=1}^{n} J_{j}\left(-\Delta_{a b} \frac{\mu_{j}}{T}\right) \\
& \sigma=J_{q, b}^{\prime}\left(\Delta_{a b} \frac{1}{T}\right)+\sum_{j=1}^{n} J_{j}\left(-\frac{\Delta_{a b} \mu_{j}\left(T_{a}\right)}{T_{a}}\right) \\
& \sigma=J_{q, a}^{\prime}\left(\Delta_{a b} \frac{1}{T}\right)+\sum_{j=1}^{n} J_{j}\left(-\frac{\Delta_{a b} \mu_{j}\left(T_{b}\right)}{T_{b}}\right)
\end{aligned}
$$

From a theoretical perspective, the three sets of fluxes and forces are equivalent. But from a practical perspective, there are some clear differences. The measurable heat flux, also known as the sensible heat flux, is a quantity that can be measured in practice. Experimental values for resistivities are always related to the measurable heat flux. The measurable and total heat fluxes are related via the partial molar enthalpies $H_{j}$ multiplied with the molar fluxes $J_{j}$ :

$J_{q}=J_{q}^{\prime}+\sum_{j=1}^{n} H_{j} J_{j}$

In practice we calculate enthalpy differences, or relative enthalpies, rather than absolute enthalpies. It is therefore very hard to link the total heat flux to practical situations. Similar to the enthalpy, we also calculate chemical potential differences rather than absolute chemical potentials. The driving forces in Eqs. (2) and (3) are therefore more practical to use than the ones in Eq. (1). These are three reasons to favour the measurable heat flux formulation over the total heat flux formulation.

The system consists of at least three control volumes: one liquid control volume, one interface control volume and one vapour control volume. It was found by Van der Ham et al. (2010) that more control volumes should be used to describe the liquid and vapour films. The driving forces and fluxes in a control volume can be gathered by a driving force vector and a flux vector. The driving force vector of each control volume is then given by the product of a resistivity matrix and the flux vector. The sum of the driving force vectors of all control volumes yields the total driving force vector of the system. If the flux vector is exactly the same in all control volumes, which means that the fluxes are constant throughout the system, it is straightforward to calculate them once the total resistivity matrix is known. The differences in chemical potential divided by temperature can be summed directly. But this is not possible for the differences in chemical potential evaluated in Eqs. (2) and (3), because they are all evaluated at different constant temperatures. At steady state, the total heat flux is constant throughout the system, similar to the molar fluxes. But the measurable heat flux is not constant. These are two reasons to favour the total heat flux formulation over the measurable heat flux formulation.

Instead of selecting one of these two formulations, a new formulation is introduced that uses the measurable heat flux at a certain reference location $J_{q, 0}^{\prime}$ and evaluates all chemical potential differences at a certain reference temperature $T_{0}$. The choice of using such reference points must be compensated for in the resistivity matrix of the control volume. Replacing the measurable heat flux at one location by the measurable heat flux at another location can be done using the energy balance shown given by Eq. (4):

$J_{q, a}^{\prime}=J_{q, b}^{\prime}+\sum_{j=1}^{n} \Delta_{a b} H_{j} J_{j}$

\subsection{Assuming constant enthalpy}

Replacing a chemical potential difference at one temperature by a chemical potential difference at another temperature requires an assumption. First, the difference in chemical potential divided by temperature is rewritten in terms of a chemical potential difference at a constant temperature $T_{a}$ :

$$
\begin{aligned}
-\Delta_{a b}\left(\frac{\mu_{j}}{T}\right)= & -\left(\frac{\mu_{j}\left(T_{b}, x_{j, b}\right)}{T_{b}}-\frac{\mu_{j}\left(T_{a}, x_{j, a}\right)}{T_{a}}\right) \\
= & -\left(\frac{\mu_{j}\left(T_{a}, x_{j, b}\right)}{T_{a}}-\frac{\mu_{j}\left(T_{a}, x_{j, a}\right)}{T_{a}}\right) \\
& +\frac{\mu_{j}\left(T_{a}, x_{j, b}\right)}{T_{a}}-\frac{\mu_{j}\left(T_{b}, x_{j, b}\right)}{T_{b}} \\
= & -\frac{\Delta_{a b} \mu_{j, T_{a}}}{T_{a}}-\int_{T_{a}}^{T_{b}} \frac{d}{d T}\left(\frac{\mu_{j, x_{j, b}}}{T}\right) d T
\end{aligned}
$$

The partial derivative of the chemical potential divided by temperature with respect to temperature is given by the Gibbs-Helmholtz equation:

$\frac{\partial}{\partial T}\left(\frac{\mu_{j}}{T}\right)=-\frac{H_{j}}{T^{2}}$

The partial enthalpy is a function of temperature. Including this temperature dependency into the equations will eventually result into third order temperature difference terms in the entropy production expression. The theory of irreversible thermodynamics only uses terms up to the second order. So in order to avoid introducing any third order term, we assume that the partial enthalpies are independent of temperature within the control volume we are considering. If we introduce Eq. (7) into Eq. (6) with this in mind we obtain:

$-\Delta_{a b}\left(\frac{\mu_{j}}{T}\right)=-\frac{\Delta_{a b} \mu_{j, T_{a}}}{T_{a}}-H_{j, x_{j, b}} \Delta_{a b} \frac{1}{T}$

Instead of going to an expression containing the chemical potential at constant temperature $T_{a}$, we can also go to an ex- 
pression at constant temperature $T_{b}$. The difference between the expressions for the two constant temperatures is the composition at which the partial enthalpy is evaluated. Eq. (8) will be used to obtain an expression for going directly from one constant temperature to another:

$$
-\frac{\Delta_{a b} \mu_{j, T_{b}}}{T_{b}}=-\frac{\Delta_{a b} \mu_{j, T_{a}}}{T_{a}}-\Delta_{a b} H_{j} \Delta_{a b} \frac{1}{T}
$$

Although the partial enthalpies are considered to be independent of temperature within the control volume, we still need to choose at which constant temperature we evaluate them. This temperature must be the same for all volumes.

\subsection{Formulae for total resistivities}

Based on Eq. (3), the following force-flux relations can be written for a single control volume:

$$
\begin{array}{r}
\Delta_{a b} \frac{1}{T}=r_{q q}^{a b} J_{q, a}^{\prime}+\sum_{j=1}^{n} r_{q j}^{a b} J_{j} \\
-\frac{\Delta_{a b} \mu_{i}\left(T_{b}\right)}{T_{b}}=r_{i q}^{a b} J_{q, a}^{\prime}+\sum_{j=1}^{n} r_{i j}^{a b} J_{j}
\end{array}
$$

Here, $r_{m n}^{a b}$ represents the resistivity in the control volume between points $a$ and $b$ that is coupling driving force $m$ with flux $n$, where $m, n \in q, i, j$. The subscript $q$ indicates the thermal driving force or flux, and the subscripts $i$ and $j$ indicate component driving forces or fluxes. The resistivity $r_{q q}$ is related to the thermal conductivity, the resistivities $r_{i j}=r_{j i}, r_{i i}$, and $r_{j j}$ are related to diffusion, and the resistivities $r_{i q}=r_{q i}$ and $r_{j q}=r_{q j}$ are related to the coupling between thermal and component fluxes, also known as the Soret and Dufour effects.

Equations (10) and (11) can be rewritten using Eqs. (5) and (9) in order to replace $J_{q, a}^{\prime}$ with the measurable heat flux at the vapour boundary of the system $\left(J_{q, v}^{\prime}\right)$ and in order to evaluate the chemical potential differences at the temperature of the liquid boundary of the system $\left(T_{l}\right)$ instead of at $T_{b}$ :

$$
\begin{array}{r}
\Delta_{a b} \frac{1}{T}=r_{q q}^{a b} J_{q, v}^{\prime}+\sum_{j=1}^{n}\left(r_{q j}^{a b}+r_{q q}^{a b} \Delta_{v b} H_{j}\right) J_{j} \\
-\frac{\Delta_{a b} \mu_{i}\left(T_{l}\right)}{T_{l}}=r_{i q}^{a b} J_{q, v}^{\prime}+\sum_{j=1}^{n}\left(r_{i j}^{a b}+r_{i q}^{a b} \Delta_{v b} H_{j}\right) J_{j} \\
+\Delta_{a b} H_{i} \Delta_{l a} \frac{1}{T}
\end{array}
$$

Kjelstrup \& De Koeijer (2003) and Bedeaux \& Kjelstrup (2004) give formulae for the total resistivities of a system that consists of three connected control volumes. It is shown by Van der Ham et al. (2010) how force-flux relations Eqs. (12) and (13) can be used to derive general formulae for the total resistivities of a system consisting of a series of $m$ connected control volumes:

$$
\begin{gathered}
r_{q q}^{t o t}=\sum_{k=1}^{m} r_{q q}^{k} \\
r_{q j}^{t o t}=r_{j q}^{t o t}=\sum_{k=1}^{m} r_{q j}^{k}+r_{q q}^{k} \Delta_{k_{v} v} H_{j}
\end{gathered}
$$

$$
\begin{gathered}
r_{i j}^{t o t}=r_{j i}^{t o t}=\sum_{k=1}^{m} r_{i j}^{k}+r_{i q}^{k} \Delta_{k_{v} v} H_{j}+r_{q j}^{k} \Delta_{k_{v} v} H_{i} \\
+r_{q q}^{k} \Delta_{k_{v} v} H_{j} \Delta_{k_{v} v} H_{i}
\end{gathered}
$$

Where $\Delta_{k_{v} v}$ indicates the difference between the vapour boundary of the system and the boundary of control volume $k$ that is closest to the system vapour boundary.

\section{Calculations}

The routine described by Van der Ham et al. (2010) allows the calculation of values for the molar fluxes and the measurable heat flux when the conditions at the liquid and vapour boundaries of the system are known. A thermodynamically consistent solution is found by requiring the entropy productions calculated using both irreversible thermodynamics and the entropy balance to be equal. This requirement is used to find the liquid film thickness for a fixed vapour film thickness. All calculations reported in this work were done using this calculation routine.

It is discussed in detail by Van der Ham et al. how to calculate all relevant resistivities. The influence of inaccuracies in their values on the calculation results was described in a sensitivity analysis. The influence of the number of control volumes per film, of the interface resistances, of the chosen vapour film thickness and of the order of the control volumes was also investigated. In the current study we always: used a minimum of 32 control volumes per film, included the interface resistances, used a vapour film thickness of $5 \times 10^{-4}$ $\mathrm{m}$ and described the system from the liquid to the vapour boundary.

\subsection{Distillation column design}

The work by Van der Ham et al. discussed the influence of coupling between thermal and mass fluxes on the transfer rates in a nitrogen-oxygen mixture at one single point in a distillation column. In this work, we investigated how this influence changes along the length of the distillation column. In order to obtain sets of boundary conditions that represent positions along the entire length of the column, we started by defining a base case distillation column design.

3.1.1 Column specifications. The distillation column design we used as base case is separating a binary nitrogenoxygen mixture with a nitrogen mole fraction of 0.80 into products with purities of 0.99 . The feed and the top product are vapours at their dew points and the bottom product is a liquid at its bubble point. It is assumed that the column operates at a constant pressure of 1.4 bar. The total number of stages is 18 , excluding reboiler and total condenser. At stage 11 , counting from top to bottom, a feed with a flow rate of $1 \mathrm{~mol} / \mathrm{s}$ enters the column. An equilibrium stage model was used to simulate the column; it was solved using the bubble point method as described by Seader \& Henley (1998). A high accuracy thermodynamic model was used to calculate the required thermodynamic properties; it is described by Lemmon et al. (2000). This model was used for all thermodynamic calculations that were done in this work.

3.1.2 Column profiles. Table 1 gives an overview of how the characteristic column properties change within the column. The temperature, liquid mole fraction $x$, vapour mole 
fraction $y$, liquid flow rate $L$ and vapour flow rate $V$ are given as function of the stage number $n$. The condenser and reboiler are represented by stage numbers $C$ and $R$.

Table 1. Column profiles of the temperature, the nitrogen mole fractions, the liquid flow and the vapour flow.

\begin{tabular}{|c|c|c|c|c|c|}
\hline $\begin{array}{l}n \\
(-)\end{array}$ & $\begin{array}{c}T \\
(\mathrm{~K})\end{array}$ & $\begin{array}{c}x_{N_{2}} \\
(-)\end{array}$ & $\begin{array}{c}y_{N_{2}} \\
(-)\end{array}$ & $\begin{array}{c}L \\
(\mathrm{~mol} / \mathrm{s})\end{array}$ & $\begin{array}{c}V \\
(\mathrm{~mol} / \mathrm{s})\end{array}$ \\
\hline$C$ & 80.28 & 0.9900 & - & 0.639 & - \\
\hline 1 & 80.45 & 0.9652 & 0.9900 & 0.634 & 1.445 \\
\hline 2 & 80.72 & 0.9289 & 0.9791 & 0.627 & 1.440 \\
\hline 3 & 81.09 & 0.8793 & 0.9633 & 0.618 & 1.433 \\
\hline 4 & 81.57 & 0.8174 & 0.9420 & 0.607 & 1.424 \\
\hline 5 & 82.13 & 0.7485 & 0.9159 & 0.595 & 1.413 \\
\hline 6 & 82.72 & 0.6812 & 0.8874 & 0.585 & 1.401 \\
\hline 7 & 83.25 & 0.6237 & 0.8602 & 0.576 & 1.391 \\
\hline 8 & 83.68 & 0.5798 & 0.8373 & 0.570 & 1.382 \\
\hline 9 & 83.99 & 0.5492 & 0.8201 & 0.565 & 1.376 \\
\hline 10 & 84.20 & 0.5291 & 0.8082 & 0.563 & 1.372 \\
\hline 11 & 84.34 & 0.5165 & 0.8005 & 0.561 & 1.369 \\
\hline 12 & 84.62 & 0.4907 & 0.7839 & 0.558 & 0.367 \\
\hline 13 & 85.23 & 0.4380 & 0.7468 & 0.551 & 0.364 \\
\hline 14 & 86.39 & 0.3478 & 0.6702 & 0.541 & 0.357 \\
\hline 15 & 88.17 & 0.2322 & 0.5363 & 0.530 & 0.347 \\
\hline 16 & 90.15 & 0.1290 & 0.3603 & 0.523 & 0.337 \\
\hline 17 & 91.71 & 0.0618 & 0.1991 & 0.519 & 0.329 \\
\hline 18 & 92.64 & 0.0265 & 0.0926 & 0.517 & 0.325 \\
\hline$R$ & 93.11 & 0.0100 & 0.0364 & 0.194 & 0.323 \\
\hline
\end{tabular}

3.1.3 Stage and point boundary conditions. The point in the distillation column that was used as base case by Van der Ham et al. was defined by calculating average temperatures and mole fractions for a certain stage. The considered stage was located around the centre of the top part of the column, which corresponds to a position in between stages 5 and 6 in the column that was used in this work. The vapour and liquid boundary conditions based on stage $n$ can be calculated using the following formula:

$T_{l}^{n, s}=1 / 2\left(T^{n-1}+T^{n}\right)$

$T_{v}^{n, s}=1 / 2\left(T^{n}+T^{n+1}\right)$

$x_{N_{2}}^{n, s}=1 / 2\left(x_{N_{2}}^{n-1}+x_{N_{2}}^{n}\right)$

$y_{N_{2}}^{n, s}=1 / 2\left(y_{N_{2}}^{n}+y_{N_{2}}^{n+1}\right)$

Instead of using boundary conditions based on average stage values, it is also possible to select boundary conditions based on the point in between two stages. In a packed column, the liquid flowing down from a stage meets the vapour rising up from the stage below at this point. These point boundary conditions are given by:

$T_{l}^{n, p}=T^{n-1}$

$T_{v}^{n, p}=T^{n}$

$x_{N_{2}}^{n, p}=x_{N_{2}}^{n-1}$

$y_{N_{2}}^{n, p}=y_{N_{2}}^{n}$

A schematic overview of stage $n$ is shown in Figure 2, including the quantities that are required to determine the boundary conditions sets for stage $n$ and for points $n$ and $n+1$.

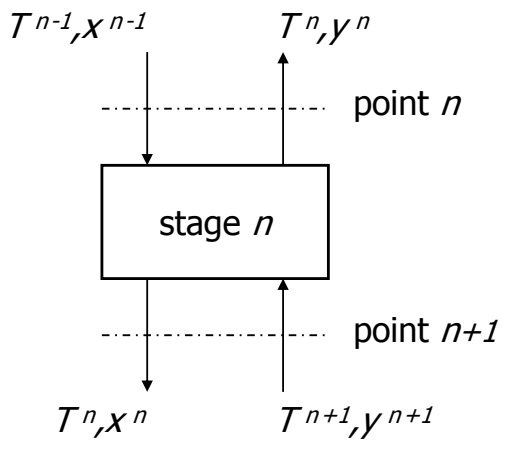

Figure 2. Schematic overview of stage $n$, depicting the quantities that are required to calculate the boundary conditions sets for stage $n$ and for points $n$ and $n+1$.

Using the column design presented in this section, it is possible to calculate 18 sets of stage boundary conditions. Because the points above stage 1 and below stage 18 can also be used as a set of boundary conditions, there exist 19 sets of point boundary conditions. When plotted as function of position in the column, each of the stage boundary conditions is located in between two point boundary conditions.

\section{Results and discussion}

\subsection{Nitrogen flux profile}

Using the temperature and mole fraction data given in Table 1 and using Eqs. (17)-(20) and (21)-(24), 37 sets of system boundary conditions have been determined; 18 sets of stage boundary conditions and 19 sets of point boundary conditions. Each of these sets has been used as input to the calculation model for coupled transfer of mass and thermal energy. Figure 3 gives an overview of the calculated nitrogen fluxes as function of position in the column.

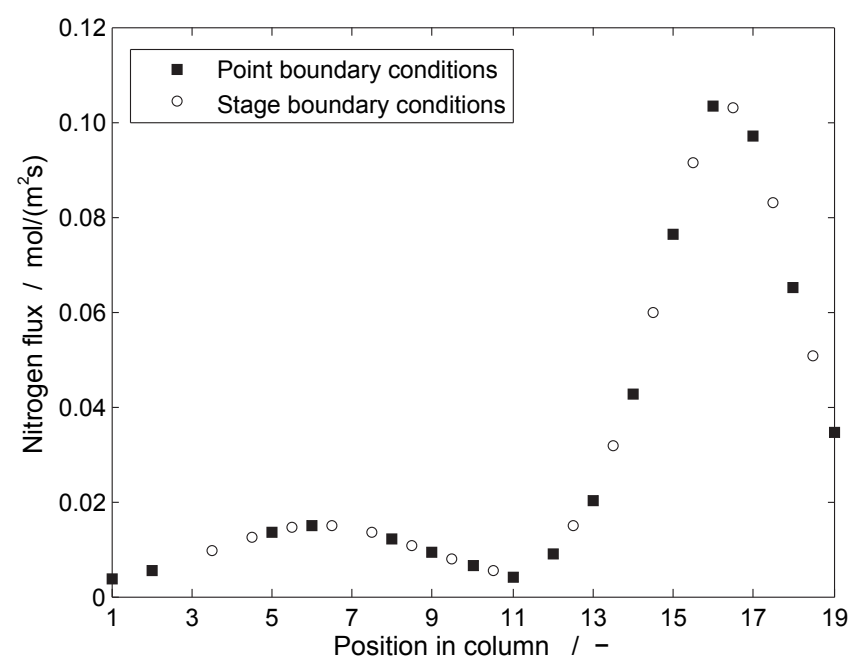

Figure 3. Comparison between the calculated nitrogen flux as function of the position in the column for both point and stage boundary conditions.

Only the profile of the nitrogen flux is shown in Figure 3; the profiles of the other fluxes and of the entropy production are comparable. Although we used 37 sets of boundary conditions as input, the figure contains only 31 data points. It 
proved impossible to find a liquid film thickness that yielded a thermodynamically consistent system for the other 6 sets of boundary conditions.

There can be different reasons why it might be impossible to solve the model for some specific cases. It might be caused by the fixed vapour film thickness we use, or it could be related to possible inaccuracies in the calculated resistivities. It can also be related to the fact that we are using boundary conditions originating from an equilibrium stage model as input to our rather rate-based like model. At the moment we do not have sufficient understanding to predict beforehand which cases are impossible to solve.

The data points from the two different types of boundary conditions are very well in agreement with each other. The combination of the two gives a good representation of how the calculated nitrogen flux changes along the entire length of the column.

\subsection{Nitrogen transfer profiles}

Based on the mole fraction and flow data in Table 1, it is possible to calculate how much nitrogen is transferred on each of the stages. It does not make any sense to make a direct comparison between the amounts of transferred nitrogen and the calculated nitrogen fluxes shown in Figure 3. The calculated fluxes are values at a single point and they are given per amount of interfacial area, while the transferred amounts are the totals of complete stages. But if we assume that the interfacial area is constant along the length of the column and express the fluxes and transferred amounts as percentages of their column averages, we can still compare how the two quantities change along the length of the column. This comparison is shown in Figure 4.

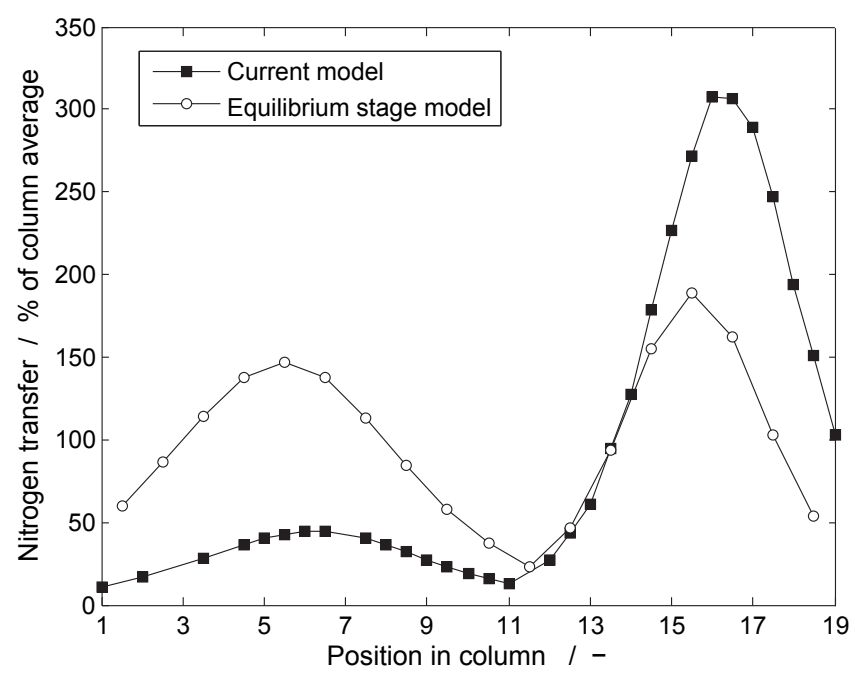

Figure 4. Comparison between the nitrogen fluxes calculated with the model presented in this work and the total amounts of transferred nitrogen calculated from the equilibrium stage model.

The comparison between the two nitrogen transfer profiles shows that they follow the same trend; both have a minimum around the feed stage and maxima around the centres of the top and bottom parts. But the relative magnitudes of the two maxima are different. The maxima are comparable in the equilibrium stage model profile. But in the profile based on the calculations done in this work, the bottom part maximum is almost seven times bigger than the top part maximum. A part of this difference can be related to the constant vapour film thickness that we have used in our calculations. An increase in the vapour film thickness would translate into an increase in the total resistivities, which means that a fixed set of boundary conditions yields smaller fluxes.

There are two reasons why we expect the vapour film thickness to be larger in the bottom part of the column. The first one is related to the vapour flow rates in the column. As can be seen in Table 1, the vapour flow rate is about four times larger in the top part of the column. A larger vapour flow rate corresponds to a larger superficial vapour velocity, which means a smaller vapour film thickness. The second reason is related to the viscosity of the vapour phase. When going from the top to the bottom of the column, the temperature and the oxygen fraction increase. Because oxygen has a higher viscosity than nitrogen and because the viscosity increases with an increasing temperature, the viscosity must be higher in the bottom part of the column. A higher vapour viscosity corresponds to a larger vapour film thickness. How much these two effects exactly affect the vapour film thickness should be investigated in future studies.

\subsection{Neglecting coupling resistances}

Similar to Van der Ham et al. (2010), we have investigated the effect of neglecting coupling between thermal and molar fluxes. This can be done by setting all control volume resistivities that couple a thermal and a molar flux equal to zero, while fixing the film thickness ratio at the value found for the coupled case. We have done this for all data points shown in Figure 3 and calculated the relative differences between the fluxes obtained from uncoupled systems and the fluxes obtained from coupled systems. Table 2 gives the averages and standard deviations of these relative differences, summarizing the effect of neglecting coupling for the entire column.

Table 2. The average and standard deviation of the influence that neglecting coupling resistances has on the different fluxes.

\begin{tabular}{lrrrr}
\hline & $J_{N_{2}}$ & $J_{O_{2}}$ & $J_{q, l}^{\prime}$ & $J_{q, v}^{\prime}$ \\
\hline Average (\%) & 11 & -4.2 & -39 & -3.3 \\
Standard deviation (\%) & 11 & 5.4 & 29 & 0.2 \\
\hline
\end{tabular}

Similar to what was found by Van der Ham et al. for a single stage, the neglect of coupling mostly affects the nitrogen flux and the measurable heat flux at the liquid boundary. The column average values are lower than the values found for the single stage investigated by Van der Ham et al.. The standard deviations in the effects on the molar fluxes and on the measurable heat flux at the liquid boundary are of the order of their averages. There is no clear relation between these effects and the position in the column. The deviations are mainly caused by deviations in the liquid film thicknesses that were found. The vapour film thickness was fixed at the same value for all systems, which partly explains the relatively constant and small effect that neglecting coupling has on the measurable heat flux at the vapour boundary. Figure 5 shows how this effect changes along the length of the column.

The effect that neglecting coupling between thermal and 


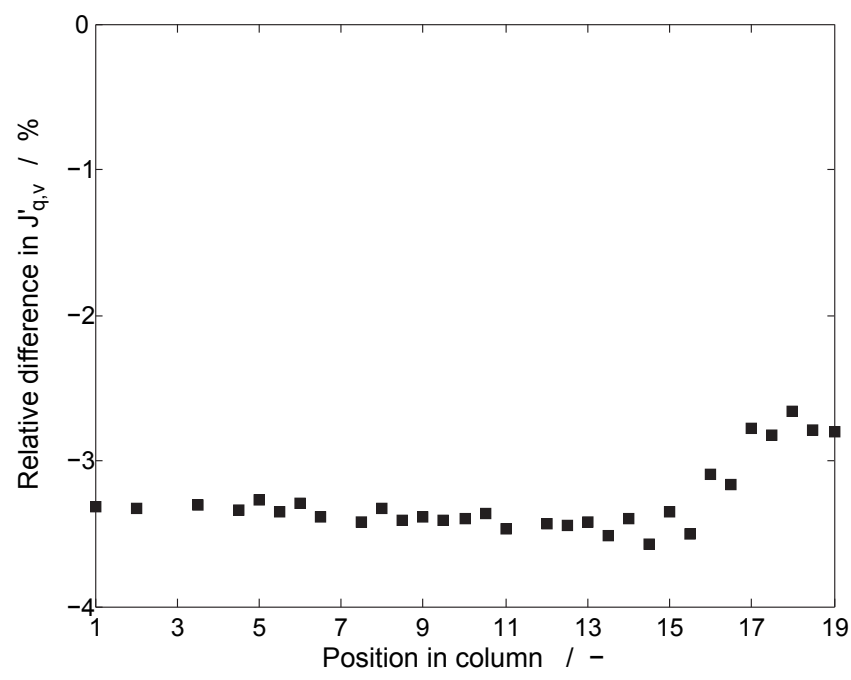

Figure 5. Effect of neglecting coupling on the measurable heat flux at the vapour boundary, as function of the position in the column.

molar fluxes has on the measurable heat flux at the vapour boundary is relatively constant along the length of the column. It increases slowly in the top part of the column, but towards the bottom of the column it starts decreasing.

The thermal and molar fluxes are central variables in the minimization of entropy production in distillation columns. It is therefore essential to have accurate models to calculate them.

\section{Conclusions}

Calculating fluxes from a combination of stage and point boundary conditions yields a consistent representation of how the fluxes vary along the length of the entire distillation column. The trend in the obtained nitrogen flux profile is similar to the one based on an equilibrium stage model, but the distribution of the nitrogen transfer over the two column halves does not agree. This disagreement can be partly explained by the use of a constant vapour film thickness. Including a dependency of the vapour film thickness on the vapour flow rate and viscosity is an important next step in the development of the model. The effect of neglecting coupling between thermal and mass fluxes on their calculated magnitudes is smaller when averaged for the entire column than it is for the single stage investigated earlier, but it is still considerable and should be considered in models used for optimization studies.

\section{Acknowledgements}

The research leading to these results has received funding from the European Community's 7th Framework Programme (FP7/2007-2013) under grant agreement number 211971 (The DECARBit project).

\section{Nomenclature}

$H_{j} \quad$ partial molar enthalpy of component $j, \mathrm{~J} / \mathrm{mol}$

$J_{j} \quad$ molar flux of component $j, \mathrm{~mol} /\left(\mathrm{s} \mathrm{m}^{2}\right)$

$J_{q} \quad$ total heat flux, $\mathrm{J} /\left(\mathrm{s} \mathrm{m}^{2}\right)$

$J_{q}^{\prime} \quad$ measurable heat flux, $\mathrm{J} /\left(\mathrm{s} \mathrm{m}^{2}\right)$

$L \quad$ liquid flow rate, $\mathrm{mol} / \mathrm{s}$

$m$ number of control volumes, dimensionless

$r_{m n}$ resistivity coupling driving force $m$ with flux $n$, where $m, n \in q, N_{2}, O_{2}$
$S_{j} \quad$ partial molar entropy of component $j, \mathrm{~J} /(\mathrm{K}$ mol $)$

$T$ temperature, $\mathrm{K}$

$V \quad$ vapour flow rate, $\mathrm{mol} / \mathrm{s}$

$x \quad$ liquid mole fraction, dimensionless

$y \quad$ vapour mole fraction, dimensionless

$\Delta_{a b} Y$ difference in property $Y: Y_{b}-Y_{a}$

Greek symbols

$\mu_{j} \quad$ chemical potential of component $j, \mathrm{~J} / \mathrm{mol}$

$\sigma \quad$ local entropy production, $\mathrm{J} /\left(\mathrm{K} \mathrm{m}^{2}\right)$

Subscripts and superscripts

$0 \quad$ reference point

$a, b \quad$ location indices

$k \quad$ control volume index

$l \quad$ liquid

$n \quad$ stage index

$\mathrm{N}_{2}$ nitrogen

$\mathrm{O}_{2}$ oxygen

$p$ point

$q$ thermal energy

$s \quad$ stage

tot total

$v \quad$ vapour

\section{References}

Bedeaux, D., \& Kjelstrup, S. (2004). Irreversible thermodynamics - a tool to describe phase trasitions far from global equilibrium. Chemical Engineering Science, 59(1), 109118.

De Koeijer, G. M., \& Kjelstrup, S. (2004). Application of Irreversible Thermodynamics to Distillation. International Journal of Thermodynamics, 7(3), 107-114.

Fonyó, Z. (1974a). Thermodynamic analysis of rectification II. Finite cascade models. International Chemical Engineering, 14, 203-210.

Fonyó, Z. (1974b). Thermodynamic analysis of rectification I. Reversible model of rectification. International Chemical Engineering, 14, 18-27.

Kjelstrup, S., \& Bedeaux, D. (2008). Non-equilibrium thermodynamics of heterogeneous systems. Singapore: World Scientific Publishing Co.

Kjelstrup, S., \& De Koeijer, G. M. (2003). Transport equations for distillation of ethanol and water from the entropy production rate. Chemical Engineering Science, 58(7), 1147-1161.

Lemmon, E. W., Jacobsen, R. T., Penoncello, S. G., \& Friend, D. G. (2000). Thermodynamic properties of air and mixtures of nitrogen, argon, and oxygen from 60 to $2000 \mathrm{~K}$ at pressures to $2000 \mathrm{MPa}$. Journal of Physical and Chemical Reference Data, 29(3), 331-385.

Seader, J. D., \& Henley, E. J. (1998). Separation process principles. New York, USA: John Wiley and Sons, Inc.

Taylor, R., \& Krishna, R. (1993). Multicomponent mass transfer. New York, USA: John Wiley and Sons, Inc.

Van der Ham, L. V., Bock, R., \& Kjelstrup, S. (2010). Modelling the coupled transfer of mass and thermal energy in the vapour-liquid region of a nitrogen-oxygen mixture. Chemical Engineering Science, 65(6), 2236-2248. 